\title{
A Comparison of Perioperative Blood Transfusions and Other Complications Following Transverse Rectus Abdominis Myocutaneous Flaps versus Deep Inferior Epigastric Perforator Flaps
}

\author{
Rajith A. Amaratunga, Bishoy Soliman, Thomas C. Lam \\ Department of Plastic and Reconstructive Surgery, Westmead Hospital, Sydney, Australia \\ Email: rajith1@live.com.au
}

How to cite this paper: Amaratunga, R.A., Soliman, B. and Lam, T.C. (2017) A Comparison of Perioperative Blood Transfusions and Other Complications Following Transverse Rectus Abdominis Myocutaneous Flaps versus Deep Inferior Epigastric Perforator Flaps. Surgical Science, 8, 145153.

https://doi.org/10.4236/ss.2017.83016

Received: February 9, 2017

Accepted: March 10, 2017

Published: March 13, 2017

Copyright $(9) 2017$ by authors and Scientific Research Publishing Inc. This work is licensed under the Creative Commons Attribution International License (CC BY 4.0).

http://creativecommons.org/licenses/by/4.0/

\begin{abstract}
Objective: Abdominal based free flaps such as the Transverse Rectus Abdominis Myocutaneous (TRAM) flap and Deep Inferior Epigastric Perforator (DIEP) flap are essential tools in the reconstructive armamentarium post mastectomy. These reconstructions are often prolonged, complex and associated with complications. This study aims to compare the complication rate, particularly focusing on perioperative transfusions, between TRAM and DIEP flaps performed by the senior author in our tertiary referral centre. Methods: A retrospective review was conducted of one hundred and seven consecutive TRAM and DIEP flaps from 2000 to 2014 . The two groups were analysed for demographics, preoperative risk factors and post-operative complications including blood transfusions, haematomas, flap losses, redo-anastomoses, flap infections, abdominal wound sequelae and non-surgical complications. Results: Sixty-three patients underwent 67 free/muscle sparing TRAM flaps and 35 patients underwent 40 DIEP flaps. There were no statistically significant differences in patient demographics or preoperative risk factors between the two groups. Five TRAM flap cases (7.9\%) required transfusion whilst no DIEP flap cases required transfusion. This difference was not found to be statistically significant $(\mathrm{p}=0.16)$. However, free/muscle-sparing TRAM flaps were found to have a significantly higher overall complication rate compared to DIEP flaps $(23.8 \%$ vs. $5.7 \%, \mathrm{p}=0.02)$. Conclusion: The current study demonstrated no difference in perioperative transfusion requirement between TRAM and DIEP cases. There was however a significantly higher rate of overall complications associated with TRAM flaps warranting the authors to conclude that care be taken when opting for this reconstructive method.
\end{abstract}




\section{Keywords}

Breast Reconstruction, DIEP, TRAM, Complications

\section{Introduction}

The use of autologous free tissue transfer for breast reconstruction post mastectomy has become a main stay of management throughout the past decade [1] [2] [3] [4] [5]. Abdominal based free flaps such as the Transverse Rectus Abdominis Myocutaneous (TRAM) flap and Deep Inferior Epigastric Perforator (DIEP) flap are essential tools in the reconstructive armamentarium post mastectomy. These are often prolonged, complex procedures which can result in more complications than other reconstructive options [6]. Research comparing the two approaches seems to indicate that each method has its strengths and weaknesses. More specifically TRAM flaps have been associated with more abdominal hernias and fat necrosis [7], longer hospital stays and worse postoperative pain [8], whilst DIEP flaps have been reported to have higher numbers of flap losses [9]. Despite an adequate level of research comparing most complication rates there has been a relative paucity of attention given to comparing perioperative transfusion rates between these two reconstructions. Individually TRAM cases have been found to have transfusion rates ranging from $6 \%$ to $95 \%$ [10]. Similarly, DIEP cases have been reported to have transfusion rates ranging from $9.1 \%$ to as high as $80.3 \%$ [11] [12].

Perioperative blood loss is a significant issue as it directly impacts flap outcomes [13]. Equally important was the finding of a recent review which highlighted the tendency for increased medical and surgical complications in free flap reconstructions that underwent blood transfusion post operatively [14]. Thus the decision to transfuse blood should not only take into consideration the scarcity of blood products and the cost to the health care system but also the potential harm to the patient. This study aims to compare the rate of complications, particularly focusing on blood transfusion rates, between free/musclesparing TRAM and DIEP flap breast reconstructions at a tertiary referral center in Sydney, Australia.

\section{Method}

A retrospective review was conducted of consecutive free/muscle-sparing TRAM and DIEP breast reconstructions performed by the senior author [TL] and his team between January 2000 and January 2014 at Westmead Hospital, a tertiary referral center in Sydney, Australia. One hundred and two cases were identified of which 4 patients were excluded as they underwent a combination of a free/ muscle-sparing TRAM and a DIEP flap for bilateral reconstructions in the single procedure leaving 98 cases for analysis.

Patient files and electronic records were reviewed for each case. Records were analysed for preoperative risk factors, post-operative complications and transfu- 
sion requirement. Risk factors analysed included age, body mass index (BMI), smoking status, prior adjuvant therapy, pre-operative haemoglobin concentration, timing and extent of reconstruction. Complications assessed included haematoma, flap loss (partial or complete), flap infections, re-do anastomoses, abdominal wound sequelae and non-surgical complications. The protocol for transfusion opted by the unit was a haemoglobin concentration less than $7 \mathrm{~g} / \mathrm{dl}$ or if patients were clinically symptomatic. A combination of unpaired $t$ tests and Fisher's exact tests were performed to assess for a difference between TRAM and DIEP groups in relation to demographics, risk factors, transfusion rate and other complication rates. A value of $\mathrm{p}<0.05$ was considered significant in all statistical analyses.

\section{Flap Choice}

Since transitioning to DIEP reconstructions in 2003, the senior author discovered zone 4 to be unreliable and with varying venous insufficiency making the choice to do DIEP more selective. TRAM flap reconstructions were opted for when patients needed large flaps especially if zone 4 was included. TRAM flaps were also chosen for patients who had previously received radiotherapy.

\section{Results}

\subsection{Demographics}

A total of 107 consecutive free/muscle-sparing TRAM and DIEP flaps were performed on 98 patients. Sixty-three patients underwent 67 free/muscle-sparing TRAM flaps and 35 patients underwent 40 DIEP flaps. The mean age of both groups was 50 years. Mean BMI was 30.0 for TRAM patients and 32.1 for DIEP patients. Thirty (47.6\%) TRAM patients and 17 (48.6\%) DIEP patients underwent preoperative adjuvant therapy. Tobacco use was reported in $22(37.3 \%)$ TRAM cases and 16 (47.1\%) DIEP cases. Four (6.3\%) TRAM cases and 5 (14.3\%) DIEP cases underwent bilateral reconstructions. Immediate reconstruction was performed on 14 (22.2\%) TRAM cases and 6 (17.1\%) DIEP cases with the remaining cases being delayed reconstructions. Mean preoperative haemoglobin concentration was $13.6 \mathrm{~g} / \mathrm{dL}$ in both TRAM and DIEP cases.

Using an unpaired $t$ test, there was no difference identified in age, BMI or preoperative haemoglobin levels between the groups. There was also no significant difference identified between the groups in exposure to adjuvant therapy, tobacco use, number of bilateral cases and immediate reconstructions using Fisher's exact tests (Table 1).

\subsection{Blood Transfusions}

A total of five cases (5.1\%) required transfusion. All five cases were TRAM flaps (7.9\% of TRAM cases). Two of these cases were transfused due to intraoperative blood loss. A further two cases were transfused after returning to theatre for evacuation of chest wall haematomas. One case required transfusion for significant post-operative blood loss following revision of a thrombosed arterial anas- 
Table 1. Patient characteristics.

\begin{tabular}{cccc}
\hline Patient & & Flap Type & \\
Characteristics & TRAM (\%) & DIEP (\%) & $p$ \\
\cline { 2 - 4 } & & & $0.91^{\mathrm{a}}$ \\
Age (Years) & 50 & 50 & \\
Mean & $44-57$ & $42-54$ & $0.84^{\mathrm{a}}$ \\
Range & & & \\
BMI (kg/m $\left.{ }^{2}\right)$ & 30.0 & 32.1 & $1.00^{\mathrm{b}}$ \\
Mean & $27-33$ & $28-36$ & $0.38^{\mathrm{b}}$ \\
Range & $30(47.6)$ & $17(48.6)$ & $0.27^{\mathrm{b}}$ \\
Adjuvant Therapy & $22(37.3)$ & $16(47.1)$ & $0.27^{\mathrm{b}}$ \\
Tobacco & $59(93.7)$ & $30(85.7)$ & $0.61^{\mathrm{b}}$ \\
Unilateral & $4(6.3)$ & $5(14.3)$ & $0.61^{\mathrm{b}}$ \\
Bilateral & $14(22.2)$ & $6(17.1)$ & $0.69^{\mathrm{a}}$ \\
Immediate & $49(77.8 \%)$ & $29(82.9 \%)$ & \\
Delayed & 136 & 136 & \\
Preoperative Hb (g/dL) & & & \\
\hline
\end{tabular}

${ }^{a}$ Unpaired $t$ Test; ${ }^{b}$ Fisher's Exact Test.

tomosis and commencement of a heparin infusion. Using the Fisher's exact test this difference in transfusion rates between TRAM and DIEP flaps was not found to be statistically significant $(\mathrm{p}=0.16)$.

Three of 78 delayed cases (3.8\%) required transfusion compared to two of 20 (10\%) immediate reconstructions. Also four out of 89 (4.5\%) unilateral reconstructions and one out of 9 (11.1\%) bilateral reconstructions underwent transfusion. These differences were also found not to be statistically significant using Fisher's exact test $(\mathrm{p}=0.27$ and $\mathrm{p}=0.39)$.

\subsection{Other Complications}

Overall, 17 cases (17.3\%) had a total of 23 complications. Fifteen (23.8\%) TRAM cases had 20 complications and two (5.7\%) DIEP cases had three complications. The TRAM flap complications included three haematomas (4.8\%), two cases of partial flap loss (3.2\%), three re-do anastomoses (4.8\%), three cases of flap infection (4.8\%), five cases with abdominal wound sequelae (7.9\%) and four cases with non-surgical complications (6.3\%). Abdominal wound sequelae in this group included one case of fat necrosis and four cases of abdominal wound infections. Cases with systemic illness included two cases of pneumonia, one case of type II respiratory failure propagated by atelectasis and one case of atrial fibrillation with recurrent rapid ventricular rate.

DIEP flap complications consisted of two (5.7\%) cases of abdominal wound sequelae and one case (2.9\%) of systemic illness. Abdominal wound issues included one case of fat necrosis and one case of infection. The non-surgical complication in this group was a patient who developed post-operative urinary retention requiring prolonged urethral catheterisation. There were no incidences of haematoma, partial flap loss, re-do anastomoses or flap infections. Using the Fisher's exact test, the higher rate of complications associated with TRAM flaps was found to be significant $(\mathrm{p}=0.02)$. There was no statistically significant dif- 
ference found for any individual complication between the two groups (Table 2).

\subsection{Operator Dependency}

A total of 70 cases $(71.4 \%)$ were performed by the senior author with the remaining 28 (28.6\%) cases being performed by his team. Three cases (4.3\%) performed by the senior author required transfusion compared to two cases $(7.1 \%)$ performed by his team. Complications arose in twelve cases (17.1\%) performed by the senior author compared to five cases $(17.9 \%)$ in those performed by his team. Using Fisher's exact test there was no statistically significant difference found for either transfusion rates or complication rates when comparing cases performed by the senior author to those performed by his team $(\mathrm{p}=0.62$ and $\mathrm{p}$ $=0.10$ respectively).

\section{Discussion}

Autologous abdominal based free flaps such as the TRAM and DIEP flaps have undoubtedly become one of the most preferred reconstructive methods for breast reconstruction [1] [2] [3] [4] [5]. TRAM and DIEP flap reconstructions are often lengthy, complex operations which can at times result in significant blood loss in the perioperative setting. In these instances blood transfusion is a necessity to maintain haemoglobin concentrations and haematocrit at an appropriate level to ensure adequate blood flow, oxygenation of tissue and subsequent free flap survival [15]. However care should be taken when considering transfusion. Along with the scarcity of blood products, the cost to the health care system and the risk of disease transmission there are also the recent findings that blood transfusions are related to increased medical and surgical complications following free flap reconstructions [6] [16].

The majority of research on transfusion rates have investigated pedicled TRAM flaps as opposed to free/muscle-sparing TRAM flaps with transfusion rates ranging from $95 \%$ in earlier studies to $2.9 \%$ in more recent times [17]. In 2014, Lee et al looked at 261 patients who underwent free TRAM flap reconstructions and found that $28.4 \%$ of cases required perioperative transfusion [10].

Table 2. Complications by type.

\begin{tabular}{cccc}
\hline & & Flap Type \\
Complications & TRAM (\%) & DIEP (\%) & $p^{a}$ \\
\cline { 2 - 3 } & & & \\
Haematoma & $3(4.8)$ & 0 & 0.55 \\
Partial Flap Loss & $2(3.2)$ & 0 & 0.54 \\
Redo-anastomosis & $3(4.8)$ & 0 & 0.55 \\
Flap infection & $3(4.8)$ & 0 & 0.55 \\
Abdominal wound Sequelae & $5(7.9)$ & $2(5.7)$ & 1.00 \\
Systemic & $4(6.3)$ & $1(2.9)$ & 0.65 \\
\hline
\end{tabular}

${ }^{a}$ Fisher's Exact Test. 
This is markedly higher than our rate of $7.9 \%$. The disparity maybe due to the larger scale of their study or their indication to transfuse being a haemoglobin value less than $8 \mathrm{~g} / \mathrm{dL}$, as opposed to less than $7 \mathrm{~g} / \mathrm{dL}$ in our study.

Similarly the DIEP flap transfusion rate of $0 \%$ in our study was lower than that reported in the literature. Appleton et al. (2011) after reviewing 144 DIEP cases reported $18.8 \%$ of patients required blood transfusion. The decision to transfuse was similar to our study in that cases were transfused if there were clinical signs of hypovolaemia or the haemoglobin was less than $7 \mathrm{~g} / \mathrm{dl}$ [18]. Likewise,Lymperopoulos et al. (2013) studied 131 patients who underwent DIEP reconstructions and found $9.1 \%$ of patients required transfusion post operatively [11]. However a study performed in Melbourne, Australia noted that up to $80.3 \%$ of DIEP cases in their study required perioperative transfusion [12]. This high number of cases may be due to the fact that some patients included in the study were preoperatively anaemic. Though the authors have not mentioned how many cases were anaemic the mean preoperative haemoglobin for the study was $11.8 \mathrm{~g} / \mathrm{dL}$, with a value less than $12.0 \mathrm{~g} / \mathrm{dL}$ deemed anaemic. The authors found a significant relationship between preoperative anemia and post-operative transfusion requirement. The current study had a mean preoperative haemoglobin of $13.6 \mathrm{~g} / \mathrm{dL}$ for both TRAM and DIEP cases with only two patients having a haemoglobin level less than $12.0 \mathrm{~g} / \mathrm{dL}$.

In the current study the difference in transfusion rates between free/musclesparing TRAM and DIEP flaps was not found to be significant. This may be a result of the small sample size having inadequate power to detect a significance. Perhaps with a larger sample size the increased rate of transfusion in TRAM flaps may have been deemed significant. If this were the case then a possible explanation may be that there is greater blood loss when harvesting TRAM flaps due to the harvesting of muscle. It is however difficult to conclude that the reason for transfusion could be ascribed to the type of flap performed in this series. Furthermore the inherent choice to perform a TRAM flap instead of a DIEP flap due to poor perforators may indicate an overall poor candidate for free flap repair which may have been complicated regardless of which flap was used. It is important to note that in addition to lower transfusion rates in the DIEP cases in our study, there were no significant systemic complications nor were their incidences of flap loss.

Of the five cases which required transfusion, three cases were unilateral delayed breast reconstructions. The remaining two were immediate reconstructions, one of which was immediate and bilateral. Though a theoretical risk exists with immediate bilateral reconstructions with a prolonged procedure time and increased risk of blood loss requiring transfusion, the current findings do not reflect this sentiment with the five transfused cases being varied in their timing and extent of reconstruction. However it is interesting to note that the immediate bilateral reconstruction case required transfusion in the absence of a complication requiring take back to theatre, alluding to the possibility of a higher risk in these prolonged procedures. Thus it may be that in the absence of any 
other complication transfusion may be avoided when unilateral reconstruction is opted for over bilateral. Though ultimately there was no significant increase in transfusion rates for bilateral or immediate reconstructions we advocate for greater care and awareness when attempting immediate bilateral reconstructions.

In relation to overall complication rate this study found TRAM flaps to have significantly more complications than DIEP flaps. This finding is in contention with the literature which has mainly found no difference in overall complication rates between the two methods [7] [19]. In their meta-analysis Wang et al (2014) found TRAM and DIEP flaps to have complication rates of $25.6 \%$ and $27.9 \%$ respectively [7]. The TRAM complication rate in the current study was similar at 23.8\% however our DIEP complication rate was lower at only 5.7\%. Furthermore they noted that TRAM flaps were associated with significantly higher rates of hernias whereas DIEP flaps were associated with more incidences of fat necrosis. These findings were not shared in the current study with no statistically significant difference found in any individual complication type between the two groups. This finding may be due to the initial learning curve experienced when TRAM reconstructions were first performed. It is likely that experience with complications arising from TRAM flap reconstructions would have aided in preventing similar events in DIEP cases. Alternately, the higher complication rate of TRAM flaps may be due to the procedure being more invasive and harvesting a larger amount of tissue than DIEP reconstructions. Also, the resultant defect is larger making primary closure more difficult and possibly leading to abdominal wound issues. Furthermore, the subsequent pain and reduced mobility may explain the increased incidence of atelectasis and pneumonia in this group compared to DIEP flaps.

The current study also analysed the data to assess for a possible operator dependency given our centre is a teaching hospital and surgeons in training often perform procedures. The majority of cases were performed by the senior author who is a Senior Consultant Surgeon in Plastic and Reconstructive Surgery. He transitioned to DIEP flap reconstructions from 2003. There was no evidence of operator dependency in regards to complications or perioperative transfusion requirement.

\section{Conclusion}

Given the association of blood transfusion with increased post-operative complications in free flap surgery it is important to determine whether one form of abdominal based reconstruction is more likely to result in blood loss requiring transfusion than the other. The current study found no difference in transfusion rates between TRAM and DIEP flap reconstructions. However TRAM flaps were found to have a higher overall complication rate than DIEP flaps. Consequently the authors advocate for greater care and awareness when opting for free/muscle-sparing TRAM reconstructions. Future research in the form of large prospective randomised trials may be better able to elucidate a difference in transfu- 
sion rates if one exists between these two work horses of the reconstruction ladder.

\section{References}

[1] Hartrampf, C.J. and Bennett, G.K. (1987) Autogenous Tissue Reconstruction in the Mastectomy Patient. A Critical Review of 300 Patients. Annals of Surgery, 205, 508519. https://doi.org/10.1097/00000658-198705000-00009

[2] Grotting, J., Urist, M., Maddox, W., et al. (1989) Conventional TRAM Flap versus Free Microsurgical TRAM Flap for Immediate Breast Reconstruction. Plastic and Reconstructive Surgery, 83, 828-841. https://doi.org/10.1097/00006534-198905000-00009

[3] Hamdi, M., Weiler-Mithoff, E. and Webster M. (1999) Deep Inferior Epigastric Perforator Flap in Breast Reconstruction: Experience with the First 50 Flaps. Plastic and Reconstructive Surgery, 103, 86-95. https://doi.org/10.1097/00006534-199901000-00015

[4] Blondeel, P.N. (1999) One Hundred Free DIEP Flap Breast Reconstructions: A Personal Experience. British Journal of Plastic Surgery, 52, 104-111. https://doi.org/10.1054/bjps.1998.3033

[5] Gill, P., Hunt, J., Guerra, A., et al. (2004) A 10-Year Retrospective Review of 758 DIEP Flaps for Breast Reconstruction. Plastic and Reconstructive Surgery, 113, 1153. https://doi.org/10.1097/01.PRS.0000110328.47206.50

[6] Fischer, J., Nelson, J., Au, A., Ct, T., Serletti, J. and Wu, L. (2014) Complications and Morbidity Following Breast Reconstruction-A Review of 16,063 Cases from the 2005-2010 NSQIP Datasets. Journal of Plastic Surgery and Hand Surgery, 48, 104-114. https://doi.org/10.3109/2000656X.2013.819003

[7] Wang, X., Liu, L., Song, F. and Wang, Q. (2014) Meta-Analysis of the Safety and Factors Contributing to Complications of MS-TRAM, DIEP, and SIEA Flaps for Breast Reconstruction. Aesthetic Plastic Surgery, 38, 681-691. https://doi.org/10.1007/s00266-014-0333-3

[8] Kroll, S., Sharma, S., Koutz, C., et al. (2001) Postoperative Morphine Requirements of Free TRAM and DIEP Flaps. Plastic and Reconstructive Surgery, 107, 338. https://doi.org/10.1097/00006534-200102000-00006

[9] Man, L.X., Selber, J.C. and Serletti, J.M. (2009) Abdominal Wall Following Free TRAM or DIEP Flap Reconstruction: A Meta-Analysis and Critical Review. Plastic and Reconstructive Surgery, 124, 752-764. https://doi.org/10.1097/PRS.0b013e31818b7533

[10] Lee, H., Kim, D., Jin, U., Jeon, Y., Hwang, J. and Park, H. (2014). Effect of Perioperative Transfusion of Old Red Blood Cells on Postoperative Complications after Free Muscle Sparing Transverse Rectus Abdominis Myocutaneous Flap Surgery for Breast Reconstruction. Microsurgery, 34, 434-438.

https://doi.org/10.1002/micr.22240

[11] Lymperopoulos, N., Sofos, S., Constantinides, J., Koshy, O. and Graham, K. (2013) Blood Loss and Transfusion Rates in DIEP Flap Breast Reconstruction. Introducing a New Predictor. Journal of Plastic and Reconstructive Aesthetic Surgery, 66, 16591664. https://doi.org/10.1016/j.bjps.2013.07.013

[12] Ting, J., Rozen, W., Le Roux, C., Ashton, M. and Garcia-Tutor, E. (2011) Predictors of Blood Transfusion in Deep Inferior Epigastric Artery Perforator Flap Breast Reconstruction. Journal of Reconstructive Microsurgery, 27, 233-238.

https://doi.org/10.1055/s-0031-1275486 
[13] Hill, J., Patel, A., Del Corral, G., et al. (2012) Preoperative Anemia Predicts Thrombosis and Free Flap Failure in Microvascular Reconstruction. Annals of Plastic Surgery, 69, 364-367. https://doi.org/10.1097/sap.0b013e31823ed606

[14] Motakef, S., Mountziaris, P., Ismail, I., Agag, R. and Patel, A. (2015) Emerging Paradigms in Perioperative Management for Microsurgical Free Tissue Transfer: Review of the Literature and Evidence-Based Guidelines. Plastic and Reconstructive Surgery, 135, 290-299. https://doi.org/10.1097/PRS.0000000000000839

[15] Sigurdsson, G. (1995) Perioperative Fluid Management in Microvascular Surgery. Journal of Reconstructive Microsurgery, 11, 57-65. https://doi.org/10.1055/s-2007-1006512

[16] Kim, B., Ver Halen, J., Mlodinow, A. and Kim, J. (2014) Intraoperative Transfusion of Packed Red Blood Cells in Microvascular Free Tissue Transfer Patients: Assessment of 30-Day Morbidity Using the NSQIP Dataset. Journal of Reconstructive Microsurgery, 30, 103-114. https://doi.org/10.1055/s-0033-1357275

[17] Tzilinis, A., Lofman, A. and Tzarnas, C. (2003) Transfusion Requirements for TRAM Flap Post Mastectomy Breast Reconstruction. Annals of Plastic Surgery, 50, 623-627. https://doi.org/10.1097/01.SAP.0000054181.58934.1E

[18] Appleton, S., Ngan, A., Kent, B. and Morris, S. (2011) Risk Factors Influencing Transfusion Rates in DIEP Flap Breast Reconstruction. Plastic and Reconstructive Surgery, 127, 1773-1782. https://doi.org/10.1097/PRS.0b013e31820cf1dd

[19] Chen, C., Halvorson, E., Disa, J., et al. (2007) Immediate Postoperative Complications in DIEP versus Free/Muscle-Sparing TRAM Flaps. Plastic and Reconstructive Surgery, 120, 1477-1482. https://doi.org/10.1097/01.prs.0000288014.76151.f7

\section{Submit or recommend next manuscript to SCIRP and we will provide best service for you:}

Accepting pre-submission inquiries through Email, Facebook, LinkedIn, Twitter, etc. A wide selection of journals (inclusive of 9 subjects, more than 200 journals) Providing 24-hour high-quality service User-friendly online submission system Fair and swift peer-review system Efficient typesetting and proofreading procedure Display of the result of downloads and visits, as well as the number of cited articles Maximum dissemination of your research work

Submit your manuscript at: http://papersubmission.scirp.org/ Or contact ss@scirp.org 